SLAC-PUB-8064

August 1999

\title{
DIRC Dreams: Research Directions for the Next Generation of Internally Reflected Imaging Counters*
}

\author{
Blair N. Ratcliff and Stefan Spanier \\ Stanford Linear Accelerator Center, Stanford University \\ Stanford, California 94309, USA
}

\begin{abstract}
Some conceptual design features of the total internally reflecting, imaging Cherenkov counter (DIRC) are described. Limits of the DIRC approach to particle identification, and a few features of alternative DIRC designs, are briefly explored.
\end{abstract}

Invited talk presented at the Third International Workshop on Ring Imaging Cherenkov Detectors

Ein-Gedi, Dead Sea, Israel

November 15-20, 1998

*Work supported by Department of Energy contract DE-AC03-76SF00515. 


\section{Introduction and Scope}

The DIRC, for Detection of Internally Reflected Cherenkov Light, is a subtype of Ring Imaging Cherenkov counter (RICH). It "inverts" the usual principle for use of light from the radiator of an imaging counter by collecting and imaging the total internally reflected light, rather than the transmitted light. In so doing, a DIRC utilizes the optical material of the radiator in two ways simultaneously. First, as a Cherenkov radiator, and second, as a light pipe for the Cherenkov light trapped in the radiator by total internal reflection. The high-reflection coefficients inherent in the total internal reflection process, and the fact that angles are conserved during reflection from a flat surface (up to a sign change) allow the photons of the ring image to be transported to a detector outside the path of the radiating particle, where they may be imaged $[1,2]$.

A number of DIRC prototypes have been constructed and tested over the past few years $[3,4,5,6]$. The first large-scale DIRC detector designed for physics is now finishing fabrication and is being tested in the $B A B A R$ detector at PEP-II [7]. This detector uses a radiator of 144-long fused silica bars $\left(1.7 \times 3.5 \times 490 \mathrm{~cm}^{3}\right)$ arrayed as a 12-sided polygon around the PEPII interaction region. These bars are coupled to an 11,000 photomultiplier tube (PMT) array through a $120 \mathrm{~cm}$ long standoff region filled with purified water. Though the performance of this device is expected to be excellent and well-matched to the particular requirements of an asymmetric B-factory like PEP-II, some design features, particularly the very long bars and the large water-coupled detection system, are less than ideal (at least aesthetically), and the overall design is rather inflexible. The basic features of this design were driven by "practical" compromises between detector performance on one hand, and availability, timeliness, risk, and cost of components on the other. In particular, although reflectively focused schemes were considered [8], the lack of any well-validated, cost-effective alternative to "standard" PMT's as the photon detector, was a determining factor in reaching this design. These considerations naturally led to a large photon detection plane, water coupling, and the long bars to bring the light to the PMT's in a region which could be shielded from the magnetic field.

During the intervening years, photon detectors have evolved, and it is interesting to look at how this evolution might affect some of the design possibilities for DIRC, and what continued developments might portend for the future. Thus, in the next sections, we ask your indulgence to suspend 
some of the practical considerations (temporarily) and explore some of the properties of other DIRC devices which might be constructed in a more ideal world. Due to space limitations, much of this discussion will be brief and on the "back of an envelope."

\section{The DIRC Principle}

The geometry of a single radiator of a reference conceptual DIRC is shown schematically in Fig. 1. Each radiator is a long, thin bar with rectangular cross section $\left[t_{x}, t_{y}\right]$. A track with velocity $\beta$ passing through the radiator with refractive index $n_{1}$ emits Cherenkov radiation in a cone around the particle trajectory. The source length of the light emitting region is the particle trajectory length in the radiating material. The angles, positions, momentum (and timing) of the track are normally provided by other detectors, primarily by a tracking device located in front of the radiator. If the index of refraction

of the radiating material $\left(n_{1}\right)$ substantially exceeds $\sqrt{2}$, and the index of the surrounding material $\left(n_{3}\right)$ is approximately one, then, for a particle close to $\beta=1$, some portion of the light will always be transported down the bar to the end. Since the radiator cross section is rectangular, angles are maintained in reflections at the surfaces of the bar (except for additional up-down/leftright ambiguities). Thus, in a perfect bar, the portion of the Cherenkov cone that lies inside the total internal reflection angle is transported undistorted down the bar to the end.

The Cherenkov photons are imaged onto a detector located outside the particle path. The variables of interest for particle identification are the Cherenkov emission angles $\left(\theta_{c}, \phi_{c}\right)$, but the directly measured quantities are typically two angles with respect to the end of the bar $\left(\alpha_{x}, \alpha_{y}\right)$. Because of the long optical delay line, the time of propagation down the bar $(t)$ is also related to a combination of the Cherenkov emission angles. A typical image in these three measured variables is shown in Fig. 2. These measured angles and time can be transformed into angles in Cherenkov emission space (up to a finite number of ambiguities). 


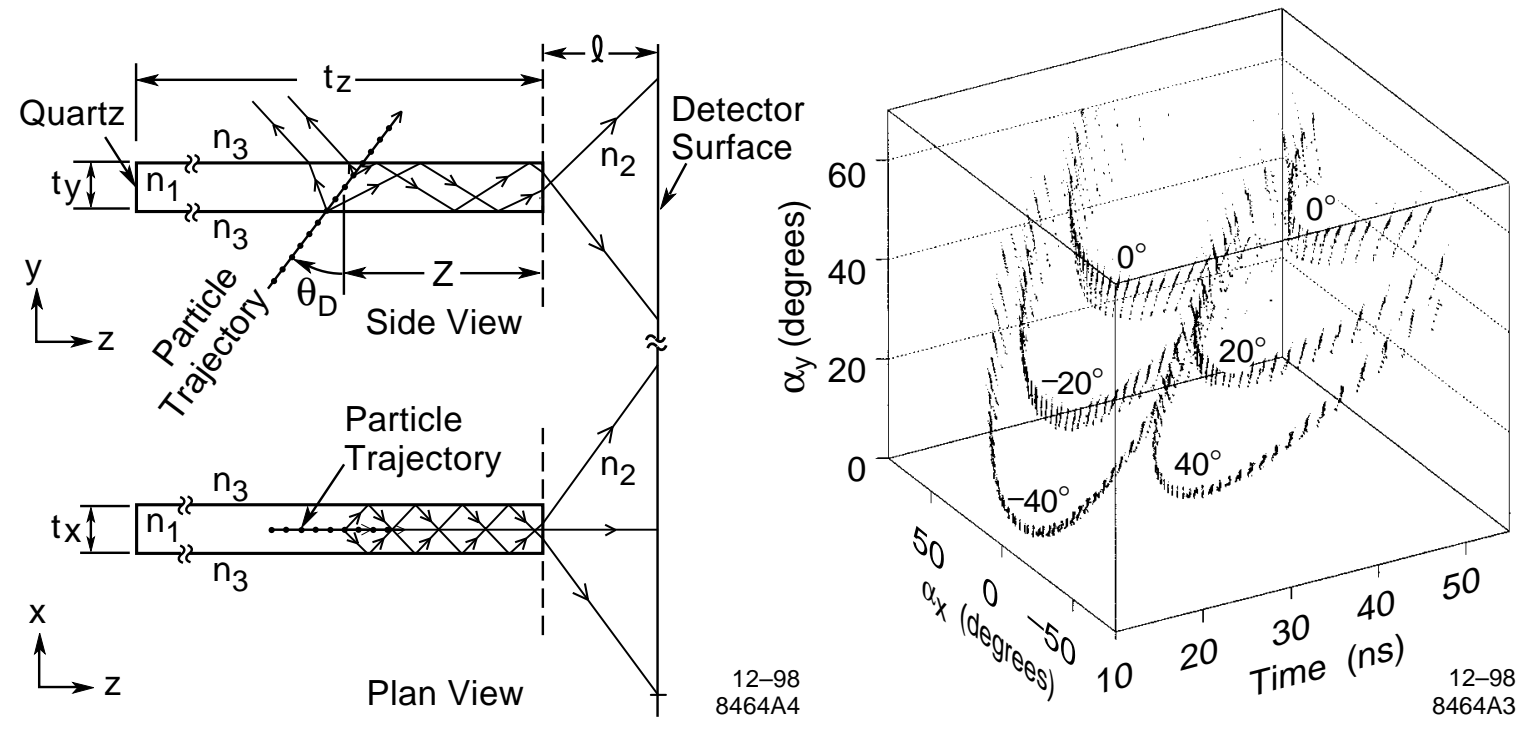

Figure 1: Schematic of a radiator bar of the DIRC counter illustrating the principle of the device. The particle trajectory is shown as a line connected by dots; representative trajectories of Cherenkov photons are shown by lines with arrows.

Figure 2: Three-dimensional images $\left(\alpha_{x}, \alpha_{y}, t\right)$ of Cherenkov photons produced by tracks at dip angles of $0^{\circ}, \pm 20^{\circ}$, and $\pm 40^{\circ}$ in a 5-m long DIRC. Measurements are made at only one bar end, with photons reflected at the other end. The image smearing is caused by chromatic dispersion in the radiator.

\section{DIRC Design Choices}

In the following sections, we will briefly review some of the choices one must make in designing a DIRC, with an eye to providing a framework for considering some possible design directions. The BaBar DIRC design provides a basic reference set of choices. Of course, there are other options and combinations possible, but only a few can be discussed in any detail. We will attempt to focus this discussion on choices that appear to be most technically feasible. 


\subsection{Cherenkov Light Production and Transport}

Fused silica is the logical material for the radiator, and all DIRC's built to date have used it. It is very transparent, has a uniform index of refraction, modest dispersion in the visible, is radiation hard, can be ground with sharp corners, and takes a good polish. Its most serious liabilities are that fused silica is expensive to procure and process, and it has a rather short radiation length $\left(X_{0}=12.3 \mathrm{~cm}\right)$. One potentially promising area to explore would be the use of plastics, particularly in an end-cap or fixed-target environment, where transmission distances can be reasonably short. This might allow a molded radiator to be made at a large savings in cost. In particular, acrylic is known to have transmission coefficients in the $10 \mathrm{~m}$ range for photon wavelengths above $400 \mathrm{~nm}[1,2,9]$. It also has fair reflection coefficients provided that the cast surfaces can be used $[9,10]$. It is inexpensive to procure and has a long radiation length $\left(X_{0}=34.4 \mathrm{~cm}\right)$, so that one can partially compensate for light loss during transmission by making the radiator thicker. However, it remains to be demonstrated whether one can obtain the index uniformity, edge sharpness, and surface and figure quality from cast surfaces to make such a detector feasible. In any case, plastic is radiation soft which will further limit potential applications.

\subsection{Cherenkov Light Transport}

The main design issue here is whether the light guide width-to-thickness ratio is such that photons reflect many times in one dimension only, or in two dimensions. This issue has been discussed previously, where the different choices were called "plates" (one dimension) and "pipes" (two dimensions) $[1,2]$. In a DIRC of the BABAR type, which uses "pipes," both transverse dimensions of the radiator bar are small and the length is long, so that the precise photon path and number of bounces are lost, or at least not utilized. The image is then essentially length independent. However, since many bounces occur from all the sides and faces, a premium is placed on the sharpness of the side/face edges, and also on the orthogonality of the angles between sides and faces. On the other hand, if one dimension of the radiator is sufficiently wide, it is possible to track the photon path from production to detection in the wide dimension. An example of such a situation is an end-cap device, (see Ref. [5]). A plate design allows the entire propagation length to be used as the standoff distance which may improve the resolution, 
and the properties of the plate sides and side/face edges become less critical. However, one must then keep track of all bounces in this side-to-side plane, and the number of ambiguities will be dependent on the length-to-width ratio. Time information may be useful to help resolve these ambiguities.

\subsection{Cherenkov Imaging (or Focusing)}

As described earlier, the direction of a photon in space is measured and then translated into a measurement of the Cherenkov angles using knowledge of the track direction. In the literature, there are shades of meaning that have been attached to the word "focusing" in the DIRC imaging process. On one hand, it has been used to refer specifically to an optical system in which light is focused onto the detector by a reflecting lens, (see, for example, Refs. $[1,2,5,8,11])$. On the other hand, it can be argued that all DIRC (indeed $\mathrm{RICH}$ ) counters must focus, in that there are no detectors in this energy regime which measure photon angles directly, and so angles must always be translated into positions by a focusing system of some kind. In this view, the differences among imaging systems are more a question of performance properties and complexity of the focusing system employed, rather than differences in basic principles. In any event, the presence of the DIRC light guide does lead to somewhat different considerations for DIRC images than is generally the case for a RICH. In particular, the methods described in Fig. 3(b) and Fig. 3(d) below are peculiar to the DIRC.

In Fig. 3, a number of different kinds of DIRC imaging systems are demonstrated schematically, along with a simple "ball park" estimate for the resolution properties of the particular systems. Imaging can be thought of as occurring separately in each of the three dimensions $(x, y$, and $t)$, and different schemes for each dimension can be used in the same counter. In principle, measurement of only two such dimensions are required in any given counter, although measurement of the third dimension is useful to reject backgrounds and ambiguities.

The analog of the classic "proximity" focusing scheme, typically used for liquid/solid radiator RICH counters, is shown in Fig. 3(a) for the DIRC case. In this scheme, the photon's angles are measured by comparing its detected position with respect to its emission point along the track. This requires knowledge of the position of the input track and the path of the photon to the detector. To use this method in the DIRC, would, in most cases, require a "plate" rather than a "pipe" for light transport. For a typical DIRC, with 


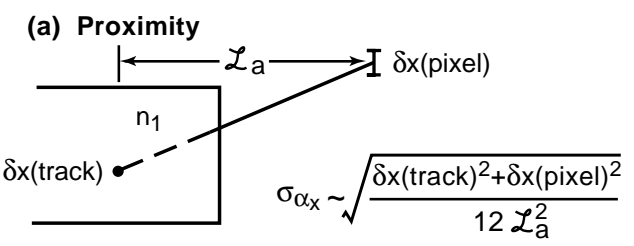

(b) Pinhole

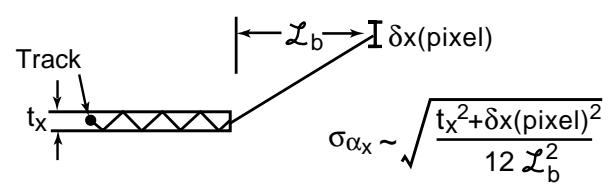

(c) Lens

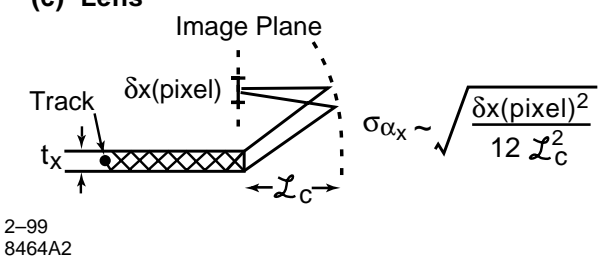

(d) Time

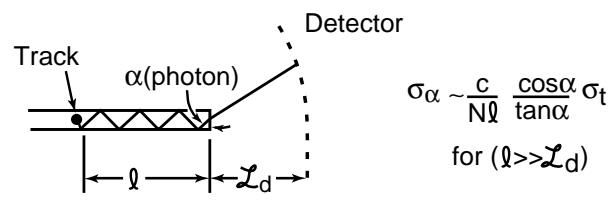

Figure 3: Schematic illustrations of four different DIRC imaging schemes: (a) proximity, (b) pinhole, (c) lens, (d) time. Simple estimates of the imaging and detector part of the resolution obtained on the photon angle in the projection shown are noted for each scheme. For simplicity, all position and detector resolutions are treated as though they are pixelized, and the indices of refraction of the Cherekov radiator and the imaging region are taken to be the same. 
rather long bars, this leads to good resolution per photon in the "proximity" focused direction. However, the price for this is the bounce ambiguities which must be resolved.

"Proximity" focusing is related to, yet subtly different than, the "pinhole" focusing method used by the BABAR DIRC, as shown in Fig. 3(b). This later imaging scheme is a direct analogue of the pinhole camera, hence the name. In this case, the path of the photon down the bar is ignored, and the resolution is independent of knowledge of track location in the bar. It does, however, depend directly on the size of the bar exit aperture. The relevant standoff length becomes the distance from the bar end to the detector plane, rather than the distance from the track to the detector plane, and may be much shorter than is the case in the "proximity" focused scheme of Fig. 3(a).

Figure 3(c) shows one version (a single reflective lens) of "lens" focusing. Other versions of lens focusing could use refractive, gradient, or diffractive lens, but the reflective system has the advantage that it allows the same material in the focusing region as in the bars, thus maximizing the overall efficiency for photon propagation. The advantage of a focusing scheme of this kind, compared to the pinhole scheme, is that the bar size can, in principle, be removed from the resolution. One can also magnify or demagnify the image to match the pixel size of a particular detector device.

Finally, Fig. 3(d) shows that the resolution of a combination of $\alpha_{x}$ and $\alpha_{y}$, the photon "dip" angle $\alpha$ (photon) with respect to the bar axis, is related to the propagation time along the bar. $^{\dagger}$ The resolution of such an angle measurement is inversely related to the distance the photon travels down the bar. This angular resolution becomes very large as the photons approach the bar axis. Thus, the resolution obtained in this method is strongly dependent on the dip angle of the track, which is correlated with the photon dip angle.

\subsection{Detectors}

The availability of detectors continues to be "the" crucial element for design. Conventional PMT's remain hard to beat for the price-to-performance ratio. In the last few years, metal channel PMT's have become available, which may be becoming competitive in cost and performance, and may allow a more elegant "lens-focused" design, although it is not quite obvious if an adequate

\footnotetext{
${ }^{\dagger}$ A device, which couples DIRC bars with a nonimaging detection system that times the first photoelectron seen at the bar end, has been proposed by Honscheid et al. [12]. A prototype has been constructed and tested by Kichimi et al. [10].
} 
packing fraction can be obtained. Hybrid PMT's look like a promising alternative for the future [13]. Other potential technologies, such as Avalanche Photodiodes [11], visible light gas detectors, VLPC's, and MCP-PMT's are more speculative. Although it is clearly difficult to imagine using a Transition Edge Sensor (TES) [14] since it requires $40 \mathrm{mK}$ temperatures, it is an exciting new high-efficiency device which has the amazing feature that it can measure the energy resolution of a visible photon to about $0.15 \mathrm{eV}$. This could reduce the chromatic term in the resolution equation by over a factor of ten (see Sec. 4.1.2 below).

\subsection{Combinations of Design Elements}

The design elements discussed above can be "mixed and matched," but only some combinations make sense. For example, the BABAR DIRC makes use of the following combination of design elements:

1. Light production: fused silica.

2. Light transport: two-dimensional (pipe).

3. Imaging principle: two-dimensional pinhole.

4. Detector: two-dimensional in space, conventional PMT, timing used to resolve ambiguities, nonmodular matching between detectors, and bars reduces pixel count.

5. Expected performance: $4 \sigma \pi-\mathrm{K}$ separation to $\sim 4 \mathrm{GeV} / \mathrm{c}$.

In contrast, suppose one would like to design an inexpensive DIRC for a medium energy fixed-target experiment. One might look for design elements something like the following:

1. Light production: acrylic.

2. Light transport: one-dimensional (plate) downstream of the target.

3. Imaging principle: proximity focused in the wide plate dimension. Onedimensional reflective focused through a molded plastic lens in the narrow plate dimension.

4. Detector: metal channel PMT with $1 \times 64$ pixels $(1 \mathrm{~cm} \times 1 \mathrm{~mm}$ pixels $)$.

5. Expected performance: $4 \sigma \pi-\mathrm{K}$ separation to $\sim 4 \mathrm{GeV} / \mathrm{c}$. 


\section{Resolution Issues}

In a simple model with equal resolution per photon, the resolution on the Cherenkov angle for a given track $\delta \theta_{c}($ tot $)$ is given by

$$
\delta \theta_{c}(t o t)=\delta \theta_{c} / \sqrt{\mathrm{N}_{\mathrm{pe}}},
$$

where $\mathrm{N}_{\mathrm{pe}}$ is the total number of photons detected, and the error on each individual photon $\delta \theta_{c}$ is given in terms of the design components discussed above as $[1,2]$

$$
\delta \theta_{c}=\sqrt{\delta \theta_{\text {Production }}^{2}+\delta \theta_{\text {Transport }}^{2}+\delta \theta_{\text {Imaging }}^{2}+\delta \theta_{\text {Detection }}^{2}},
$$

and the angular resolution on the projected track $\left(\delta \alpha_{\text {Track }}\right)$ is assumed to be sufficiently good $\left[\delta \alpha_{\text {Track }} \ll \delta \theta_{c}(\right.$ tot $\left.)\right]$ that it does not contribute significantly to the overall resolution.

The production piece $\left(\delta \theta_{\text {Production }}\right)$ is dominated by the chromatic term $\left(\delta \theta_{\text {Chromatic }}\right)$ which sets the fundamental limit for DIRC performance and limits the attainable momentum range of separation. The value ( $\left.\delta \theta_{\text {Chromatic }}\right)$ is about $5.4 \mathrm{mrad}$ for the $B A B A R$ DIRC detector.

The transport smearing $\delta \theta_{\text {Transport }}$ is caused by various flaws in the DIRC radiators, such as nonparallel sides, nonplanar surfaces, and nonorthogonal sides and faces. Due to effects of these kinds, the resolution contribution from this term tends to grow as the square root of the propagation length. In BABAR DIRC, the most difficult effect of this kind to control has been the side-to-face orthogonality, which contributes around 2-4 mrad per photon for a bar at the production specification limit. In principle, such effects can be made much smaller with different production methodology (at a higher cost), or by using a one-dimensional transport design to limit the number of side bounces.

The space part of the detector resolution is set by the pixel size coupled with the standoff distance, while the angular resolution coming from the time part is set by the length of the light propagation and the time resolution. In principle, angular resolution derived from positional information as reflected in $\delta \theta_{\text {Imaging }}$ and $\delta \theta_{\text {Detection }}$ can be made arbitrarily good to match requirements for a particular performance limit. In particular, the imaging component can be made small with lens focusing, and the number of pixels and the standoff distance are arbitrary choices. It makes economic sense to choose configurations that balance the various resolution components. 
Excellent time resolution is required to contribute in a meaningful way to DIRC angular resolution. For example, for a $B A B A R$ DIRC, one would need a time resolution of about 200 ps per photon to be competitive for a track at a zero degree track dip angle, and about three to four times better resolution to be competitive at a track dip angle around $40^{\circ}$, where many photons travel nearly along the bar axis. Another view of the effect of time resolution is shown by the example of Fig. 4, which shows the overall resolution per photon obtainable with a variety of different resolutions on the time and space variables. Similar plots for other combinations of resolutions (not shown here) demonstrate that a combination of good time resolution and one space variable can lead to rather good performance over a major portion of the phase space. This provides an interesting design possibility for a detector and focusing system with very different pixel sizes and focusing properties in the two dimensions.

\subsection{DIRC Performance Limits}

For a $\beta \approx 1$ particle of momentum $(p)$ entering a radiator with index of refraction $(n)$, the number of sigma's separation $\left(N_{\sigma}\right)$ between particles of mass $\left(m_{1}\right)$ and $\left(m_{2}\right)$ is approximately

$$
N_{\sigma} \approx\left(m_{1}^{2}-m_{2}^{2}\right) /\left(2 p^{2} \sqrt{n^{2}-1} \delta \theta_{c}(\text { tot })\right) .
$$

The large index of refraction in a DIRC radiator limits its region of good performance from low and medium momenta. In particular, $4 \sigma$ separation between pions and kaons requires a resolution on $\delta \theta_{c}($ tot $)$ of about $1.5 \mathrm{mrad}$ at $4 \mathrm{GeV} / \mathrm{c}$, and about $0.25 \mathrm{mrad}$ at $10 \mathrm{GeV} / \mathrm{c}$.

The fundamental limits on the DIRC technique are primarily (1) $N_{p e}$, (2) the chromatic smearing $\delta \theta_{\text {Chromatic }}$, and perhaps less fundamentally, (3) systematic limits on bar production quality and alignment. In the following, we briefly review how one might mitigate each of the first two components.

\subsubsection{Photon Statistics}

In principle, solid state detectors could attain two to three times the photon detection efficiency of typical PMT's. In itself, this increase in statistics leads to a rather modest improvement in the momentum range since the resolution only improves as the square root of the number of photons. In particular, with the 5.4 mrad per photon chromatic smearing of a fused silica radiator, 

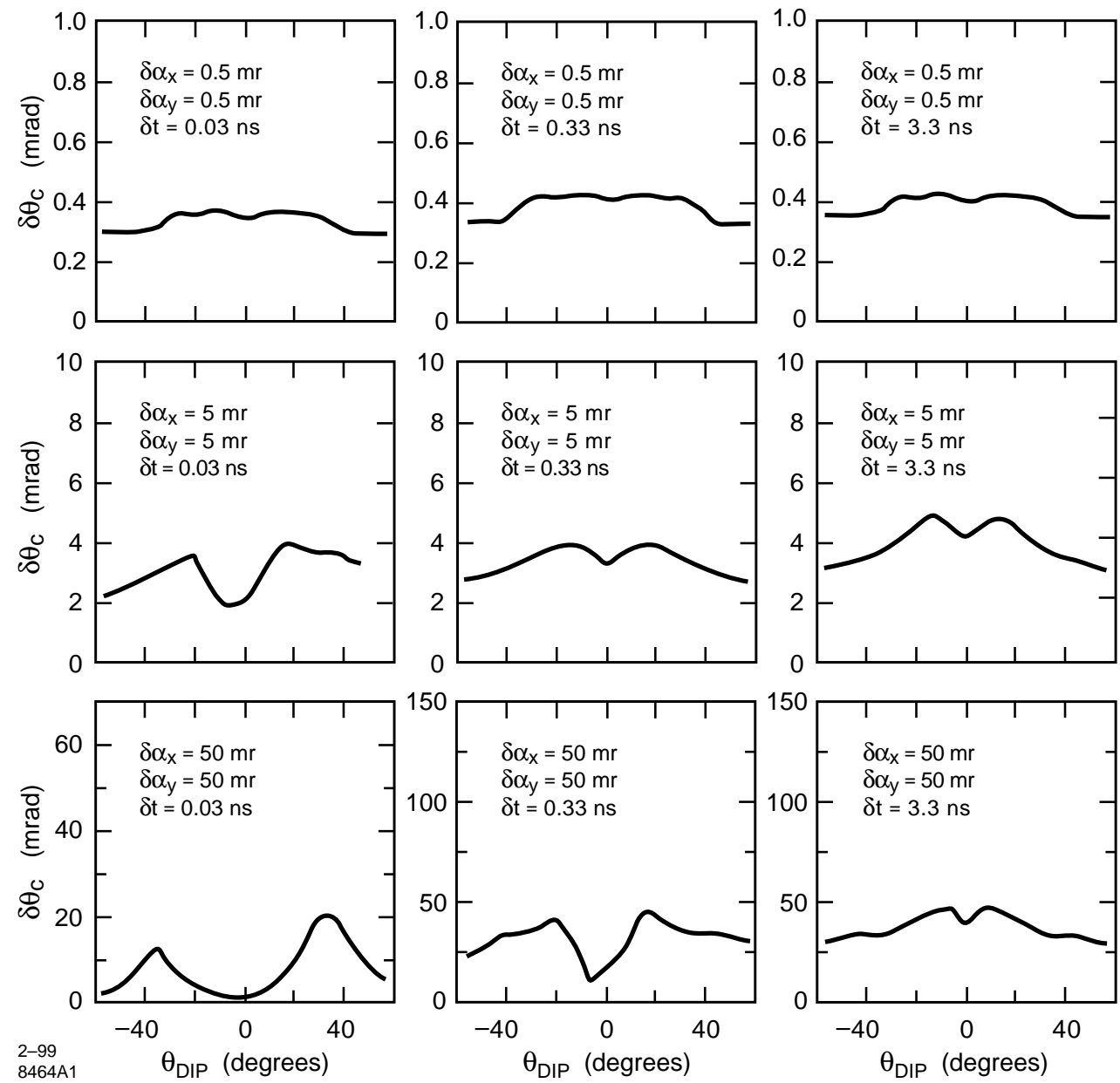

Figure 4: Resolution obtained per detected photon in a detector with bar dimensions like those of the BABAR DIRC, for a number of different resolutions on the measured photon angles and time. $\theta_{D i p}$ is the track dip angle in degrees. 
working with a bi-alkali-photocathode with a borosilicate window, it will be impractical to separate pions and kaons much above $6 \mathrm{GeV} / \mathrm{c}$, even with more than 100 photoelectrons per track.

\subsubsection{Mitigating Chromatic Smearing}

It seems impractical to apply chromatic correction in the focusing system, or to find a radiator with significantly less dispersion than fused silica. However, the high efficiency of a solid state detector could allow one to reduce the energy range of accepted Cherenkov photons. Somewhat counter-intuitively, this can actually improve the resolution substantially if the chromatic term dominates, assuming that there are enough photons to measure the remaining terms with sufficient accuracy. For example, the total resolution on a track from the chromatic term is about a factor of 2.5 better when the photon energy range is restricted between 600 and $450 \mathrm{~nm}$ compared to the case where all photons between $600 \mathrm{~nm}$ and $300 \mathrm{~nm}$ are accepted, even though the number of photons observed is reduced by nearly a factor of four! The "ideal" solution to the chromatic smearing would, of course, be a detector which measures the photon energy directly, such as the TES device described above.

\section{Summary}

DIRC's are robust, fast particle identification devices, well-suited to highradiation environments. Data from the first generation BABAR DIRC are expected in 1999. The design of elegant second generation DIRC's for new experiments depends mostly on the availability of appropriate photon detectors. Though such detectors would allow more flexible designs, the momentum range of application for DIRC's is inherently limited. The "natural" momentum separation range for good pion and kaon separation in a DIRC is up to $4-5 \mathrm{GeV} / \mathrm{c}$. It is plausible, but quite difficult, to increase this range by another factor of about two. 


\section{References}

[1] B. Ratcliff, BABAR Note 92, and SLAC-PUB-6047 (1993), published in Tsukuba B Factories, 331-341 (1992).

[2] P. Coyle et al., Nucl. Instrum. and Methods A 343, 292 (1994).

[3] D. Aston et al., IEEE Trans. Nucl. Sci. 42, 534-538 (1995) and SLACPUB-6731 (1994).

[4] C. Lu et al., Nuclear Instrum. and Methods A 371, 82-86 (1996).

[5] T. Kamae et al., Nuclear Instrum. and Methods A 382, 430-440 (1996).

[6] H. Staengle et al., Nuclear Instrum. and Methods A 397, 261-282 (1997).

[7] See, for example, the report to this conference by M. Pripstein et al., and the BABAR Collaboration, "BABAR Technical Design Report," 40, SLACR-95-457 (1995).

[8] See, for example, G. Lynch and J. Veillet, private communication.

[9] G. Kettenring, Nuclear Instrum. and Methods 131, 451-456 (1975).

[10] Kichimi et al., Nucl. Instrum. Methods A 371, 306-310 (1994).

[11] R. Wilson, report to this conference.

[12] K. Honscheid, M. Selen, and M. Sivertz, Nucl. Instrum. Methods A 343. 306-310 (1994).

[13] A. Go, report to this conference.

[14] B. Cabrera et al., Applied Physics Letters 73, 735-737 (1998). 